Consensus through Respect: A Model of Rational Group Decision-Making

Author(s): Carl Wagner

Source: Philosophical Studies: An International Journal for Philosophy in the Analytic

Tradition, Vol. 34, No. 4 (Nov., 1978), pp. 335-349

Published by: Springer

Stable URL: http://www.jstor.org/stable/4319260

Accessed: 09/05/2011 10:25

Your use of the JSTOR archive indicates your acceptance of JSTOR's Terms and Conditions of Use, available at http://www.jstor.org/page/info/about/policies/terms.jsp. JSTOR's Terms and Conditions of Use provides, in part, that unless you have obtained prior permission, you may not download an entire issue of a journal or multiple copies of articles, and you may use content in the JSTOR archive only for your personal, non-commercial use.

Please contact the publisher regarding any further use of this work. Publisher contact information may be obtained at http://www.jstor.org/action/showPublisher?publisherCode=springer.

Each copy of any part of a JSTOR transmission must contain the same copyright notice that appears on the screen or printed page of such transmission.

JSTOR is a not-for-profit service that helps scholars, researchers, and students discover, use, and build upon a wide range of content in a trusted digital archive. We use information technology and tools to increase productivity and facilitate new forms of scholarship. For more information about JSTOR, please contact support@jstor.org. 


\title{
CONSENSUS THROUGH RESPECT: A MODEL OF RATIONAL
}

\section{GROUP DECISION-MAKING}

\author{
(Received 18 November, 1977)
}

\section{INTRODUCTION}

Decision-making groups frequently face the task of reaching consensus regarding the value of one or more numerical variables. These values may represent the final outcome of deliberation (as in the case of an allocation of funds among competing projects), or they may play an intermediate role in the analysis of a decision problem (as in the case of probability and utility estimates required for computation of expected utilities). At the outset of the deliberative process there will typically be disagreement about the values in question, and thus it is of interest to develop both descriptive and normative models for the process by which consensus is, or may be, achieved.

The first steps toward the development of a descriptive model appear in French [1] and Harary [3]. Initial individual opinions are entered in a matrix $A=\left(a_{i j}\right)$, where $a_{i j}$ represents individual $i$ 's opinion as to the most appropriate value of variable $j$. This matrix is regarded as undergoing modification through repeated multiplication by a 'power matrix' $P=\left(p_{i j}\right)$, where $p_{i j}$ is a numerical measure of the power of individual $j$ to influence the opinion of individual $i$. According to this model, individuals revise their opinions over discrete units of time, holding fast (in a temporary unstable equilibrium) to their latest opinions until the forces quantified in the power matrix once again move them closer to consensus. The mathematical theory of Markov chains is used to identify a particular class of power relationships which result in convergence of opinion. Results here depend strongly upon the assumption that the power parameters $p_{i j}$ derive from a more or less enduring relationship between individuals, and thus remain constant over time. Individuals may, in fact, be unaware of the exact values of these parameters, or if aware, resentful of them.

In recent years Lehrer $[5,6,7]$ has resurrected the use of Markov chains as a model of rational decision-making by communities of distinterested experts. Here, by contrast, individuals freely and consciously grant weight to the opinions of others on the sole basis of respect for their expertise and 
sensitivity. In its present form, Lehrer's model is based on the same underlying mathematical structure as that employed by French and Harary. Initial individual opinions are registered in a matrix which undergoes consensual revision through repeated multiplication by a fixed 'respect matrix'. As presently conceived, this matrix incorporates individuals' evaluations of their colleagues relative to an entire hierarchy of judgmental skills. A physicist, for example, will receive from his colleagues a single summary weight as a scientist, a judge of scientists, a judge of judge of scientists, etc. Convinced of the desirability of refining this model to incorporate subtler analyses of the parameters of respect, Lehrer posed to me the mathematical problem of taking specific account of the different levels of respect. While the present article is intended primarily as sequel to his papers on rational social choice, it is entirely possible that the refined normative model presented here may also be of value to those interested in descriptive approaches to group decision-making. ${ }^{1}$

\section{PRELIMINARIES}

In practice, group consensus typically emerges from an unstructured discussion in which individuals modify their initial opinions on the basis of a complex set of considerations. Such discussion will involve exchange not only of indisputable facts and inferences, but also of interpretations, intuitions, and guesses which cannot be supported by rigorous logical or statistical arguments. A group under no compulsion to come to an immediate decision might, of course, exclude such impressionistic data from consideration, and decline to conclude their deliberations unless enough hard data emerges to settle the issue. But groups unable to afford the luxury of additional research may be forced to evaluate softer kinds of data and to incorporate such evaluations into their decision. Here, attention does (and should) shift from a consideration of the data to an evaluation of the individual who advocates its cogency. If such considerations have appeared to some as an unjustifiable intrusion into any truly rational decision-making process, it is perhaps because they have been employed in unsystematic ways. Thus it is of particular interest to develop systematic theories of group decision-making in which the respect accorded members of the group by each other plays an explicit role in the achievement of consensus.

Lehrer [7] has investigated two such theories, each based on the same 
underlying mathematical structure. In both, the starting point is an $n \times m$ matrix $A=\left(a_{i j}\right)$, where $a_{i j}$ represents individual $i$ 's opinion as to the most appropriate value of variable $j$ (we suppose that there are $n$ individuals and $m$ variables). Individuals are assumed to have arrived at these values after a complete discussion, so that the matrix $A$ represents what is termed a state of 'dialectical equilibrium'. In the face of disagreement at this stage, members of the group construct, after full discussion, an $n \times n$ matrix $P=\left(p_{i j}\right)$ of nonnegative real numbers with $p_{i 1}+p_{i 2}+\ldots+p_{i n}=1$ for all $i=i, 2, \ldots, n$. The entry $p_{i j}$ expresses the weight which individual $i$ grants to individual $j$ (and hence to values in the $j$ th row of $A$ ) on the basis of his respect for $j$ 's expertise and sensitivity, and may be regarded as measuring $i$ 's estimate of the chance that $j$ 's opinion $a_{j}$ is, in the relevant sense, correct. Two methods are proposed for employing this additional 'social information':

Method $I$. Each individual $i$ revises his opinion, $a_{i j}$, of the most appropriate value of variable $j$ to $a_{i j}^{(1)}=p_{i 1} a_{1 j}+p_{i 2} a_{2 j}+\cdots+p_{i n} a_{n j}$, averaging his own initial opinion with those of his colleagues according to the weighting scheme he has contributed to $P$. The resulting $n \times m$ matrix of revised values, $A^{(1)}=$ $\left(a_{i j}^{(1)}\right)$, which is simply the matrix product $P A$, may have identical rows. In such a case, consensus obtains. If not, the above process is iterated, yielding the sequence $A^{(1)}=P A, A^{(2)}=P A^{(1)}=P(P A), A^{(3)}=P A^{(2)}=P(P(P A))$, etc. By associativity of matrix multiplication, it follows that $A^{(n)}=P^{n} A$. Even if none of the matrices $A^{(n)}$ exhibits consensus, the matrices $P^{n}$ may converge (as $n$ approaches infinity) to a matrix $P^{*}$ with identical rows. (The theory of Markov chains guarantees that this will be the case, for example, when all entries of $P$ are strictly positive.) If each row of $P^{*}$ has entries $p_{1}, p_{2}, \ldots, p_{n}$, then $A^{(n)}$ converges to the consensus matrix $A^{*}=P^{*} A$. Every entry in the $j$ th column of $A^{*}$ is equal to the consensual weighted average $p_{1} a_{\mathrm{i} j}+p_{2} a_{2 j}+$ $\cdots+p_{n} a_{n j}$, and this number represents the group's consensus as to the most appropriate value of variable $j$.

The foregoing is essentially the descriptive model of French and Harary recast in normative terms. As such it requires additional rationalization of the iteration procedure employed. If the entries of $P$ are parameters of power, experience suggests that iteration may at least roughly approximate the repeated episodes of resistance and acquiescence involved in everyday group decision-making (although there are obviously situations where the parameters of power change, even radically, over time). But repeated multiplication by a matrix of weights freely chosen does not appear to be as compel- 
ling. Assuming that the move from $A$ to $P A$ is unobjectionable (since individuals construct $P$ with precisely this move in mind), one may argue that, once it is carried out, individuals have granted to each others' opinions all of the additional weight which they intended. Hence further multiplication by $P$ is gratuitious. It is asserted in [7] that an individual who rejects further iteration is repudiating the weights which he has granted to others and, in effect, giving himself unit weight and the others no weight. Such an individual will respond that he is doing nothing of the kind, pointing to his acceptance of the move from $A$ to $P A$, and protesting that he has cooperated in the construction of $P$, only to discover that it is $P^{*}$ which is to be employed in weighted averaging. Thus it seems clear that a rationalization of iteration will require that $P$ express social information of a more complex kind.

Method II. The above procedure yields, almost as an incidental byproduct, the sequence $p_{1}, p_{2}, \ldots, p_{n}$ of consensual weights. This suggests that attention be focused on determining such a sequence in an acceptable way. From this point of view the group's first task is to resolve any departure from consensus in the matrix $P$ : Suppose that in furnishing weights for the construction of $P$ individuals are asked to take into account their respect for others not only as evaluators of the numerical variable in question, but also as judges of such expertise (and, indeed, as judges of judges, etc.). Then the $i-j$ th entry of $P P, p_{i 1} p_{1 j}+p_{i 2} p_{2 j}+\cdots+p_{i n} p_{n j}$, may be interpreted as individual $i$ 's revised evaluation of individual $j$ as a variable evaluator, for it is a weighted average of all opinions $\left(p_{1 j}, p_{2 j}, \ldots, p_{n j}\right)$ on this matter, with weights $\left(p_{i 1}\right.$, $p_{i 2}, \ldots, p_{i n}$ ) determined by $i$ 's estimates of himself and his colleagues as judges of variable evaluators. Failure of consensus in $P P$ is attributed to failure of consensus in the lefmost matrix of this product, and the group multiplies this matrix on the left by $P$, thereby revising their estimates of each other as judges of variable evaluators according to their estimates of each other as judges of judges of variable evaluators.

Iteration of this procedure yields the sequence $P, P P=P^{2},(P P) P=P^{3}$, $((P P) P) P=P^{4}$, etc. The increasing powers of $P$ represent repeated attempts to resolve the lack of consensus in $P$. Should $P$ be such that these powers converge to a limit consensus matrix $P^{*}$, then any of the (identical) rows of $P^{*}$ will furnish a consensual set of weights $p_{1}, \ldots, p_{n}$ with which to average the columns of $A$.

The mathematical structures underlying Methods I and II are, of course, identical. But the conceptual differences between the techniques are signi- 
ficant, for Method II takes specific account of the complex hierarchy of social information. Indeed, a group employing the second method on a given initial matrix $A$ may arrive at a different consensus than they would have using the first method. For the broader considerations involved in constructing $P$ under Method II may yield a matrix of weights different from that which would have been chosen under Method I.

While Method II provides stronger justification for the kind of iteration it employs, it remains defective in several respects. First (and this applies to Method I as well), one may argue, opinions of a group in dialectical equilibrium have already been influenced by the patterns of respect quantified in $P$. Individuals have adopted the interpretations, intuitions, and guesses of others to the extent warranted by their respect. Hence granting any additional weight constitutes a distortion. In addition, individuals using Method II face the delicate task of incorporating into $P$ their respect for others relative to a theoretically infinite hierarchy of judgmental skills. If one is convinced, say, that good physicists are always equally good judges of physicists (and judges of judges of physicists, etc.) one may not object to employing the single matrix $P$ under the theoretically infinite number of interpretations required by Method II. But a method which aims at maximal sensitivity to all relevant information should, as Lehrer has suggested, furnish subtler analyses of the parameters of respect. In the following section we present a refinement of Method II designed to answer the above criticisms.

\section{THE METHOD OF PROXY MATRICES}

If one considers the process by which groups typically arrive at a state of dialectical equilibrium, one may quite reasonably conclude that multiplication of $A$ by any other matrix constitutes an unwarranted double weighting of information about the expertise of members of the group. For such information is almost inevitably taken into account during the discussion preceding arrival at dialectical equilibrium. Clearly, it is only by restructuring that discussion process that one can defend modification of the matrix $A$.

Fortunately, there is a way of doing this which is not merely an unobjectionable modification of ordinary practice but, in some respects, an actual improvement upon it. We simply stipulate that discussion shall take place through repeated exchange of anonymous position papers. These papers will contain everything that would be involved in a face-to-face discussion. But individuals will of necessity evaluate the opinions of others without 
taking into account the identity of the authors. They will thus delay final evaluation of the more impressionistic assertions of others, having at this point incomplete criteria for such an evaluation. When dialectical equilibrium is reached, the authors of the position papers are identified. If consensus fails, individuals turn their attention to construction of an $n \times n$ 'proxy matrix' $P_{1}=\left(p_{i j}^{(1)}\right)$, where $p_{i j}^{(1)}$ denotes that part of a unit vote which individual $i$ is willing to grant to individual $j$ on the basis of his respect for $j$ as an evaluator of the variables in question. In determining $p_{i j}^{(1)} i$ employs past information about $j$, including $j$ 's recent 'performance' as an author of position papers, to judge how much weight to grant to $j$ 's previously unweighted interpretations, intuitions, and guesses. The aim is, of course, consensus in $P_{1}$ in the form of identical rows. To that end, we envisage exchange of another round of anonymous position papers, devoted this time to a discussion of the most appropriate weights to be assigned to individuals in the decision-making group. Suppose that in dialectical equilibrium, $P_{1}$ is a consensus matrix. Each individual $i$ then agrees that the same fixed sequence $p_{1}, p_{2}, \ldots, p_{n}$ represents his proxy scheme. How will individual $i$ then revise his original estimate, $a_{i j}$, of variable $j$ ? He will reasonably attribute the difference $a_{k j}-a_{i j}$ between his opinion and that of individual $k$ to a difference in (previously unweighted) interpretations, intuitions, and guesses, and weight this difference by $p_{k}$. He will thus modify his original opinion to

$$
\begin{aligned}
a_{i j}^{(1)} & =a_{i j}+\sum_{k \neq i} p_{k}\left(a_{k j}-a_{i j}\right)=\left(1-\sum_{k \neq i} p_{k}\right) a_{i j}+\sum_{k \neq i} p_{k} a_{k j} \\
& =p_{i} a_{i j}+\sum_{k \neq i} p_{k} a_{k j}=p_{1} a_{1 j}+p_{2} a_{2 j}+\cdots+p_{n} a_{n j} .
\end{aligned}
$$

This number, which is independent of $i$, is the group consensus regarding the most appropriate value of variable $j$.

Should consensus fail for $P_{1}$ in dialectical equilibrium, the group is informed of the authors of the most recent set of position papers, and there is an exchange of anonymous position papers regarding construction of a proxy matrix $P_{2}=\left(p_{i j}^{(2)}\right)$. The number $p_{i j}^{(2)}$ represents that part of a unit vote which $i$ is willing to proxy to $j$ in the determination of $P_{1}$, and is based on $i$ 's estimate of $j$ as a contributor to that enterprise. If consensus obtains in $P_{2}$, the group replaces $P_{1}$ by $P_{2} P_{1}$, which will also be a consensus matrix (see Section 4), and derives from it, as above, consensual weighted averages of the columns of $A$. Each failure of consensus results in an attempt to resolve disagreement through the additional information contained in a proxy matrix 
of higher order. Thus, at least in theory, the group may construct an infinite sequence $P_{1}, P_{2}, P_{3}, \ldots$ of proxy matrices in its search for consensus. The sequence of products $P_{1}, P_{2} P_{1},\left(P_{3} P_{2}\right) P_{1},\left(\left(P_{4} P_{3}\right) P_{2}\right) P_{1}$ represent repeated attempts to find a consensus matrix with which to average the columns of the initial matrix $A$. Even if consensus fails in each $P_{i}$, the sequence of products may be converging to a consensus matrix. Before turning to the mathematics of convergence, however, we wish to make some general observations about the foregoing technique.

By stipulating that dicussion take place through exchange of anonymous position papers, the revised method answers the objection that any weighted averaging of the columns of $A$ is redundant. For exclusively new information is introduced in each proxy matrix. In addition, the procedure envisioned allows individuals the freedom to evaluate the opinions of others initially without being influenced by knowledge of the source of such opinions. This insures equally careful attention to the hard data presented by all individuals, thus maximizing the recognition of intersubjectively verifiable information.

Each proxy matrix, moreover, captures a particular kind of information about the judgmental skills of members of the decisionmaking group. Nothing precludes, say, an individual's receiving substantial weight as a physicist, but substantially less weight as a judge of physicists. Finally, by a careful partitioning of the task of weighing individual opinions, we have been able to furnish a clearer derivation of the weighted averages employed in earlier analyses.

\section{CONVERGENCE TO CONSENSUS: THE MATHEMATICAL THEORY}

Construction of a matrix $P_{k}$ requires each individual to determine what part of a unit vote he deems it appropriate to proxy to other individuals. His decision is circumscribed by the requirement that $P_{k}$ have unitary row sums (a kind of 'one man - one vote' restriction) and by the functional meaning of the entries in $P_{k}$. It may nevertheless be difficult for an individual to choose an actual sequence of proxy numbers, for these numbers represent personalistic evaluations rather than measurements of strictly defined phenomena. Lehrer [7] has suggested that individuals might employ as a conceptual aid in this task the following version of the classical decision theoretic device of von NeumannMorgenstern bets: An individual first decides upon a preferential ordering (with indifference allowed) of all members of the group. He assigns all 
members of the most preferred class unit weight, and all members of the least preferred class zero weight. He assigns as a weight to any other individual the unique number $q(0<q<1)$ such that he is indifferent between (1) having that individual dictate $P_{k-1}$ (or $A$, if $P_{1}$ is being constructed) and (2) a gamble in which with probability $q$ (respectively, $1-q$ ) a member of the most preferred (respectively, least preferred) class will dictated that choice. Finally, weights are normalized (by division by the sum of the weights of all individuals) so that the sum of the normalized weights is unitary.

The above method has the undesirable feature that all members of the least preferred class receive a final weight of zero, even if the individual assigning weights only slightly prefers those in the first class to those in the last as decisionmakers. While somewhat more complicated, the following generalization of that method seems desirable as an option for an individual who feels that the first method is insufficiently sensitive to his depth of preference: He first chooses nonnegative numbers $M$ and $L$ to be assigned respectively to members of the most preferred and least preferred classes. The number $M$ (respectively, $L$ ) may be thought of as an abstract value associated with having a member of the most preferred (respectively, least preferred class) dictate the decision in question. The actual values of $M$ and $L$ are immaterial, except that the ratio $M / L$ should express his best estimate of the 'ratio of preferability' of members of the first class to those of the latter class. For any other individual, he determines $q$ as above, but assigns the weight $(1-q) L+q M$, the 'expected value' of having that individual dictate the decision in question. Weights are normalized, as before, resulting in a sequence if proxy numbers, ratios among which are identical to the ratios among the prenormalized weights from which these numbers are derived.

We turn now from the construction of proxy matrices to some mathematical observations about their products. We recall that a proxy matrix is a square matrix with nonnegative entries and unitary row sums, and that a consensus matrix is any matrix with identical rows. A typical decision-making problem generates an initial $n \times m$ matrix $A$ and a sequence $P_{1}, P_{2}, \ldots$ of $n \times n$ proxy matrices. Denote by $A^{(k)}$ the product $P_{k} P_{k-1} \ldots P_{1} A$. We wish to discover conditions under which $A^{(k)}$ will converge to a consensus matrix.

Several simple preliminary observations are of interest. First, it is easy to check that if $Q$ is any consensus matrix with $n$ rows and $P$ is any $n \times n$ proxy matrix, then $P Q=Q$. Thus consensus, once achieved, is undisturbed by subsequent multiplication by proxy matrices. If, for some $k, A^{(k)}$ is a con- 
sensus matrix, construction of higher order proxy matrices is superfluous. This is, in fact, intuitively obvious, for if all agree on the assignment of a value to a particular variable, votes, however they are proxied, will all be cast on behalf of that value.

Second, if $P$ is an $n \times n$ consensus proxy matrix and $Q$ is any matrix with $n$ rows, then $P Q$ is a consensus matrix. For if the rows of $P$ are identical, then the entries in the $j$ th column of $P Q$ will be identically equal to the same weighted average of the entries in the $j$ th column of $Q$. Thus if for some $k, P_{k}$ is a consensus matrix, then $A^{(k)}=P_{k} A^{(k-1)}$ is a consensus matrix. The presence of a consensus matrix $P_{k}$ among the proxy matrices $P_{1}, P_{2}, \ldots$ is thus sufficient to guarantee that $A^{(k)}$ is a consensus matrix. Interestingly, this condition is not necessary. Suppose that

$$
A=\begin{array}{llll}
10 & 20 & 20 & 40 \\
20 & 10 & 40 & 20 \\
15 & 15 & 30 & 30
\end{array}
$$

and

$$
P_{1}=\begin{array}{lll}
0.4 & 0.2 & 0.4 \\
0.6 & 0.4 & 0 \\
0.5 & 0.3 & 0.2
\end{array}
$$

For the sake of concreteness we may suppose that the rows of $A$ represent, in thousands of dollars, allocations of 90000 dollars by three foundation directors to four grant applicants. $P_{1}$ is the directors' first order proxy matrix. Then, as may easily be checked,

$$
P_{1} A=\begin{array}{llll}
14 & 16 & 28 & 32 \\
14 & 16 & 28 & 32 \\
14 & 16 & 28 & 32
\end{array}
$$

although neither $P_{1}$ nor $A$ is a consensus matrix. Numerous examples of this phenomenon may be constructed, indicating the possibility of unanticipated sources of consensus. Thus groups using the proxy method will routinely check $P_{k} P_{k-1} \ldots P_{1} A$ for consensus before attempting to resolve disagreement in $P_{k}$ by a move to higher order proxy matrices.

Computation of the product $A^{(k)}=P_{k} P_{k-1} \ldots P_{1} A$ at each stage is also desirable from another standpoint. For suppose that divergence of opinion in 
$A^{(k)}$, as measured by the distance between the largest and smallest entries in each column, is uniformly bounded by some agreeably small positive constant $\epsilon$. Then for all $j$, we have $M_{j}-m_{j} \leqslant \epsilon$, where $M_{j}$ denotes the largest entry in column $j$ and $m_{j}$ the smallest. Moreover, if $P_{k+1}$ is any proxy matrix and $\bar{M}_{j}$ (respectively $\bar{m}_{j}$ ) denotes the largest (respectively, smallest) entry in the $j$ th column of $A^{(k+1)}=P_{k+1} A^{(k)}$, then it is easy to check that $m_{j} \leqslant \bar{m}_{j} \leqslant \bar{M}_{j} \leqslant M_{j}$. Hence if what might be termed ' $\epsilon$-consensus' obtains in some $A^{(k)}$, and if the group agrees that such approximate consensus is good enough, further multiplication by proxy matrices is superfluous. ${ }^{2}$. The group may at this point adopt the convention of taking arithmetic means of the entries in the columns of $A^{(k)}$ in order to assign final consensual values to the variables in question.

The foregoing remarks are of practical significance to a group which agrees that $\epsilon$-consensus, for some fixed $\epsilon>0$, is good enough. It is of theoretical interest, however, to characterize those sequences $P_{1}, P_{2}, \ldots$ of proxy matrices having the property that for every matrix $A$ and every $\epsilon>0$, there is some $k$ (which may depend on $A$ and $\epsilon$ ) such that $A^{(k)}=P_{k} P_{k-1} \ldots P_{1} A$ is an $\epsilon$-consensus matrix. For such proxy sequences reflect patterns of respect which ensure 'convergence to consensus'. It is fairly clear that a complete characterization of such sequences (in any terms other than those of an unenlightening paraphrase) is unlikely to be found. There is, however, a broadly applicable condition on $P_{1}, P_{2}, \ldots$ which is sufficient to guarantee that the sequence $P_{1}, P_{2} P_{1}, P_{3} P_{2} P_{1}$ converges to a consensus matrix (and, hence, that the sequence $A, A^{(1)}=P_{1} A, A^{(2)}=P_{2} P_{1} A, \ldots$ converges to consensus.) A square matrix with nonnegative entries and unitary row sums is called regular if some power of that matrix has exclusively nonzero entries. A classical result from the theory of finite Markov chains states that if $P$ is a regular matrix, then increasing powers of $P$ converge to a consensus matrix. ${ }^{3}$ Thus if the proxy matrices $P_{1}, P_{2}, \ldots$ are identically equal to some regular matrix. $P$, as in earlier models, group opinion will converge to consensus. This condition is, however, considerably stronger than necessary. For it is in fact the case (although this does not appear to be widely known ${ }^{4}$ ) that mere convergence of the sequence $P_{1}, P_{2}, \ldots$ to a regular limit matrix $P$ is sufficient to ensure that the sequence of products $P_{1}, P_{2} P_{1}, P_{3} P_{2} P_{1}, \ldots$ converges to a consensus limit matrix. For if $P$ is regular, there is a natural number $d$ such that $P^{d}$ has exclusively nonzero entires. ${ }^{5}$ Since the $P_{i}$ converge to $P$, there is some $k$ for which $i>k$ implies that $P_{i}$ will have nonzero entries at every loca- 
tion where $P$ has nonzero entries. Hence beyond $k$, products of successive blocks of $d P_{i}$ 's will be matrices with exclusively nonzero entries. It can then be shown that there is a fixed positive lower bound on these entries, from which convergence to consensus follows by the standard Markov chain argument. ${ }^{6}$ Since there are regular $n \times n$ matrices with as few as $n+1$ positive entries, ${ }^{7}$ it is clear that patterns of rather minimal respect among individuals can nevertheless yield consensus by the proxy method.

\section{SOME FURTHER REFINEMENTS}

Products of proxy matrices represent an improvement upon powers of a single respect matrix with respect to sensitivity to different levels of judgmental skills. But even proxy matrices appear to collapse complex considerations to a single 'score'. An individual involved, say, in the allocation of research funds to a number of grant applicants may, it seems, have good rational reasons for weighing a colleague's evaluations of certain applicants more heavily than that colleague's evaluations of other applicants. But the method, as presently conceived, applies the weight $p_{i j}^{(1)}$ which $i$ grants to $j$ in $P_{1}$ uniformly to $j$ 's evaluations (i.e., to every entry in the $j$ th row of $A$ ). Now if the variables corresponding to the columns of $A$ are unrelated, the group can simply seek consensus in $A$ one column at a time, employing a (possibly) different sequence of proxy matrices for each of these decision problems.

It is often the case, however, that the variables in question are related by a constraint on their sum. Allocations from a fixed budget to several competing projects, and probability estimates for a class of mutually exclusive, exhaustive events, are cases in point. If the group achieves consensus in such cases one variable at a time, the consensual values of the variables in question may sum to a value which falls short of or exceeds the required sum. (It is easy to construct examples of each of these phenomena.) Suppose that the numbers $a_{1}, a_{2}, \ldots, a_{k}$ denote these consensual values, and the sum of these numbers differs from $s$, the sum required by the constraints of the problem.

One might argue that a number $a_{i}$ represents, at any rate, the group consensus as to the relative share of $s$ to be allocated to variable $i$. It is then a simple matter to normalize the sequence $a_{1}, a_{2}, \ldots, a_{k}$ to the sequence $\bar{a}_{1}, \bar{a}_{2}, \ldots, \bar{a}_{k}$, where $\bar{a}_{i}=a_{i} s / a_{1}+a_{2}+\ldots+a_{k}$, producing thereby an allocation which satisfies the operative constraint.

This may be an unobjectionable way of proceeding in certain kinds of 
decision problems. For problems involving variables which represent probability estimates, however, this gambit appears to be rather ad hoc. Indeed, it is our contention that one ought not to use it at all. For the single 'score' $p_{i j}^{(1)}$ (or $p_{i j}^{(k)}$ ) applied to every entry in the $j$ th row of $A$ (or of $P_{k-1}$ ) neglects distinctions of the type envisioned to a much lesser degree than initial impressions might suggest. Individuals with particular expertise in evaluating certain of the variables under consideration will, afterall, largely manifest that expertise and have it taken into account during the exchange of position papers. Weights are granted to the more impressionistic data at hand and, in the case of allocation problems, reflect estimates of an individual's sense of balance and proportion with respect to the full set of variables in question. Assertions to the effect that an individual is 'good' on certain variables and 'bad' on others (in the sense that his numerical estimates of certain variables are more appropriate than his estimates of other variables) are in fact not entirely coherent. If, for example, an individual is 'good' on all but one of the variables in an allocation problem, he will by default be 'good' on the one remaining variable, for he will allocate to that variable what remains after appropriate allocations to the other variables. A variable-byvariable allocation employing distinct proxy sequences allows a decisionmaking group to ignore this simple fact.

One feels nevertheless that there is something to the claim that individuals in a decision-making group may be 'good' on some things and 'bad' on others, even within the context of constructing a single matrix. While we reject the application of such judgments with respect to individuals' evaluations of single variables, they are meaningful and deserving of consideration in the context of an individual's determination of the full set of variables with respect to different criteria. To see how such considerations may be incorporated into a decision-making process using the techniques at hand, let us consider the problem of allocating funds from a fixed sum, $s$, to $m$ competing projects. The $n$ individuals involved in this task identify a set of $r$ mutually exclusive and exhaustive criteria upon which to evaluate the projects. These criteria will vary considerably with the problem at hand. They may be imposed by external guidelines, or chosen by the decision-making group (in which case any criterion which any individual feels is relevant will be included). Instead of constructing a single $n \times m$ allocation matrix $A$, the individuals construct $n n \times m$ matrices $A_{1}, A_{2}, \ldots, A_{r}$, where the $i$-jth entry of $A_{t}$ denotes individual $i$ 's best estimate of that part of the total funds which should be 
allocated to project $j$, if criterion $t$ were the sole consideration involved. The group then works to modify each of the matrices $A_{t}$ to a consensus matrix, employing the method of proxy matrices. Different sequences of proxy matrices may arise for each of these problems, reflecting the realistic fact that an individual may be quite expert, say, at evaluating projects based on the criterion of potential ecological value, and somewhat less expert at an evaluation based on the criterion of potential value in alleviating unemploy. ment.

Suppose that the group reaches agreement in the form of a sequence $A_{1}^{*}$, $A_{2}^{*}, \ldots A_{r}^{*}$ of $n \times m$ consensus matrices. Denote by $a(t, j)$ the consensual allocation to project $j$ based solely on criterion $t$ (so that for each $t$, we have $a(t, 1)+a(t, 2)+\ldots+a(t, k)=s)$. The individuals now turn their attention to deciding upon a consensual sequence $w_{1}, w_{2}, \ldots w_{r}$ (with $0 \leqslant w_{i} \leqslant 1$ and $w_{1}+w_{2}+\ldots w_{r}=1$ ) of weights to attach to the $r$ criteria employed above. In some decision-making situations these weights may be imposed by external guidelines. If not, the group proceeds in the usual way, constructing first an $n \times r$ matrix $W=\left(w_{i t}\right)$, where $0 \leqslant w_{i t} \leqslant 1, w_{i 1}+w_{i 2}+\cdots+w_{i r}=1$ for all $i$, and $w_{i t}$ denotes individual $i$ 's best estimate of the weight which should be given to criterion $t$. In the face of disagreement, the method of proxy matrices is used in an attempt to achieve consensus. It consensual weights $w_{1}, w_{2}, \ldots, w_{r}$ emerge, then, for each $j$, the weighted average $a_{j}=w_{1} a(1, j)+w_{2} a(2, j)+$ $\cdots+w_{r} a(r, j)$ represents the group's final allocation to project $j$. (Note that $a_{1}+\cdots+a_{k}=s$.) We remark that if a decision-making task involves estimating the probability of some event $j$, then the above method simply amounts to (1) identifying a class of $r$ mutually exclusive, exhaustive events, (2) estimating their probabilities $w_{1}, w_{2}, \ldots, w_{r}$, and the conditional probabilities $a(1, j)$, $a(2, j), \ldots, a(r, j)$ of event $j$, given each of these events, and (3) combining this information in the standard way to yield the estimate, $a_{j}$, of the unconditional probability of that event.

The above method, which is often informally followed in practice, is clearly a superior way of incorporating information about the differential abilities of an individual with respect to decision-making at some fixed level. While we illustrated the application of this method to the search for consensus regarding values of the primary variables in a decision problem, it is obviously applicable to the construction of proxy matrices as well. Thus instead of constructing a single proxy matrix $P_{1}$ in which individuals rank each others' skill at evaluating the primary variables according, say, to a criterion of 
potential ecological value, the group might construct a sequence of such matrices, each capturing information about some component aspect of this skill (such as expertise in biology or chemistry, or talent in quantitative or systems analysis). Consensual values in these matrices would then be averaged by a set of weights expressing the group consensus as to the relative importance of these constitutive skills to the overall enterprise. In theory the group may choose this approach to constructing a proxy matrix at any level, but it is likely in practice not to be employed beyond the level of second order proxy matrices.

We mentioned briefly above that the set of criteria upon which a decisionmaking group makes determinations of the variables in question, as well as the weights to be granted to those criteria, might be stipulated by some other group. In such situations, one has in effect a larger decision-making group partitioned into two classes. Individuals in the first class choose the weighted evaluation criteria, and are thus responsible for 'values'. Those in the second class assign numbers to the variables in question according to these criteria, and are thus responsible for 'facts'.

This partitioning of deliberative responsibilities is a particularly appropriate way to approach public policy questions which involve scientific considerations. Citizen groups or their representatives determine the weighted criteria by which alternative courses of action are to be judged, and groups of experts provide quantitative analyses of the alternatives according to these criteria. ${ }^{8}$ May we even hope for time when even citizen groups, through the practice of rational public choice in less adversary modes, attain that level of community idealized in the proxy model?

Department of Mathematics, The University of Tennessee

\section{NOTES}

1 I first became acquainted with Lehrer's work on rational social choice at the Institute on Freedom and Causality (Center for Advanced Study in the Behavioral Sciences, Summer 1977), and am greatly indebted to him for his assistance and encouragement in the preparation of this article. I also wish to thank Institute participants Lita Furby, Hugh McCann, and Paul Sutton for helpful comments and criticism.

2 Readers interested in mathematical details may confirm that if $A=\left(a_{i j}\right)$ is an $n \times m$ matrix with $\alpha=\max \left\{\left|a_{i j}\right|\right\}$, and if $P_{k}$ is an $\epsilon / n \alpha$-consensus matrix, then $A^{(k)}=P_{k} P_{k-1} \cdots$ $P_{1} A$ will be an $\epsilon$-consensus matrix.

3 Kemeny and Snell [4], p. 70. 
4 The case of 'backwards' products $P_{1} P_{2} \ldots P_{k}$ (which are the natural generalization of powers $P^{k}$ when the $P_{i}$ are transition matrices of a nonhomogeneous Markov process) is treated in Seneta [8].

5 It is an interesting mathematical fact that the smallest $d$ for which this is the case never exceeds $n^{2}-2 n+2$. See Seneta [8] for a proof of this theorem.

6 See Kemeny and Snell [4], pp. 69-71.

7 The matrix, $P$ with rows (from first to last) $(0100),(0010),(0001)$, and $(q, 1-q, 0$, 0 ), with $0<q<1$, and the obvious $n$-dimensional generalizations of $P$, are all regular (and furnish examples to show that the upper bound $n^{2}-2 n+2$ mentioned in Note 5 is sharp. See Seneta [8]). See also Lehrer [7] for examples of regular matrices, more likely to occur in practice, in which each individual grants positive respect only to himself and to one other individual.

8 A strikingly impressive application of this approach to resolve a municipal controversy about the use of 'dum-dum' bullets by police is described by Hammond and Adelman in $[2]$.

\section{BIBLIOGRAPHY}

[1] French, John R. P., Jr.: 1956, 'A formal theory of social power', Psychological Review 63,pp. 181-194.

[ $\rightarrow$ Hammond, K. R. and Adelman, L.: 1976, 'Science, values, and human judgment', Science 194, pp. 389-396.

[3] Harary, F.: 1959, 'A criterion for unanimity in French's theory of social power', in Studies in Social Power, ed. by D. Cartwright (Institute for Social Research, Ann Arbor, Mich.), pp. 168-182.

[4] Kemeny, John G. and Snell, J. Laurie: 1976, Finite Markov Chains (Springer-Verlag, New York).

$[\rightarrow$ Lehrer, Keith: 1975, 'Social consensus and rational agnoiology', Synthese 31, pp. $141-160$.

$[\rightarrow$ Lehrer, Keith: 1976, 'When rational disagreement is impossible', Noûs 10, pp. 327-332.

[7] Lehrer, Keith: 'Consensus and comparison: A theory of social rationality', to appear.

[8] Seneta, Eugene: 1973, Nonnegative Matrices (John Wiley and Sons, New York). 